\title{
Smartphone application as an aid in determination of caries risk and prevention: A pilot study
}

\author{
Veerale Panchal ${ }^{1}$, Deepa Gurunathan ${ }^{1}$, A. K. Shanmugaavel ${ }^{1}$
}

Correspondence: Dr. Veerale Panchal Email: veerale60193@gmail.com
'Department of Paediatric and Preventive Dentistry, Saveetha Dental College, Saveetha University, Chennai, Tamil Nadu, India

\section{ABSTRACT}

Objective: To assess the dietary and oral hygiene pattern before and after the usage of cariometer app. Materials and Methods: Dietary score recorded by cariometer was assessed for 7 days for preschool children in the age group of 2-6 years. The frequency of brushing and mouth rinsing was assessed before and after the usage of app. Paired $t$-test was conducted to compare the dietary score, brushing frequency, and rinsing frequency pre and post the use of the app. Results: There was a significant improvement in the dietary pattern followed by the patients at the day 7 as compared to day 1 . About $90 \%$ of children brushed twice at day 7 of the use of cariometer. There was a significant increase in the frequency of rinsing after meals at day 7 as compared to day 1 of the use of cariometer. Conclusion: There was a significant improvement in the dietary pattern and the oral hygiene habit after the use of app.

Key words: Diet score, oral hygiene, tooth brushing

\section{INTRODUCTION}

The prevalence of dental caries in India among preschool children varies from $19.2 \%$ to $71.1 \% .^{[1]}$ However, according to the National Oral Health Survey 2002-2003 documented an average prevalence of $40.5 \%$ in the state of Tamil Nadu and $40 \%-60 \%$ in the country. ${ }^{[1]}$ According to the National Oral Health Survey 2002-2003, the caries risk for children aged 2-5 years was higher by $2 \% .{ }^{[2]}$

According to the $\mathrm{WHO}$, the multifactorial nature of dental caries is related to the presence of microorganisms, fermentable carbohydrates in the diet, particularly when consumed frequently, susceptible teeth, and early stage of development. In the causation of the caries, there is a dynamic balance between the caries risk factors and protective factors. ${ }^{[3]}$ This balance is maintained by the continuous remineralization and demineralization process which maintains the integrity of the tooth structure. ${ }^{[4]}$

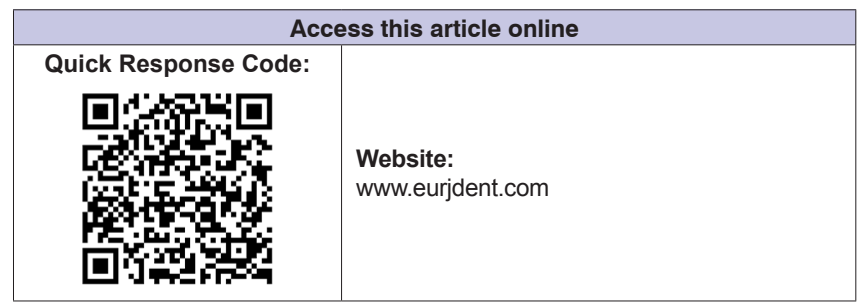

Stephan curve demonstrates the variation in $\mathrm{pH}$ of oral cavity which is a result of the acid produced by the bacteria and buffering capacity of saliva. ${ }^{[5]}$ Repeated exposure of the tooth $\mathrm{pH}$ below the critical $\mathrm{pH}$ level (5.5) ${ }^{[5]}$ increases the rate of demineralization due to the lack of calcium and phosphate. Thus, maintaining the $\mathrm{pH}$ of the oral cavity above the critical $\mathrm{pH}$ plays an important role in the prevention of demineralization. ${ }^{[5]}$

Dietary factors and food choices affect the oral $\mathrm{pH}$, thereby being important determinants in causation of dental caries. ${ }^{[6,7]}$ Parent's cultural norms is vital in influencing the child's dietary habits ${ }^{[8]}$ as well as with the changing ethnicity, there is more predisposition to consumption of fast food that leads to the increase in the consumption of cariogenic food. ${ }^{[8]}$ The association of dental caries to excessive sugar intake

This is an open access article distributed under the terms of the Creative Commons Attribution-NonCommercial-ShareAlike 3.0 License, which allows others to remix, tweak, and build upon the work non-commercially, as long as the author is credited and the new creations are licensed under the identical terms.

For reprints contact: reprints@medknow.com

How to cite this article: Panchal V, Gurunathan D, Shanmugaavel AK. Smartphone application as an aid in determination of caries risk and prevention: A pilot study. Eur J Dent 2017;11:469-74.

DOI: 10.4103/ejd.ejd_190_17 
has been affirmed by an expert panel of the World Health Organization, whose members reviewed the strength of evidence linking dietary factors to caries in 2003. ${ }^{[2]}$ The panel reported the increased risk of caries associated with frequent and total intake of free simple sugars. It also reported a decrease in the risk of caries related to consumption of hard cheeses and use of sugar-free chewing gum. It is also observed that mouth rinsing after cariogenic food consumption has a protective effect against dental caries. ${ }^{[6,7]}$ Another study by Díaz-Garrido et al. showed more frequent sucrose consumption increases the cariogenicity of Streptococcus mutans biofilm. ${ }^{[9]}$ Another study by Manju et al. demonstrated a decrease in S. mutans level with supplementary oral hygiene measures along with tooth brushing. ${ }^{[10]}$

Dietary counseling is the primordial level in the prevention of dental caries caused due to dietary influences. Together with the use of fluoride, basic rinsing practice and reducing the frequency of intake of sugar are essential for caries prevention. ${ }^{[11,12]}$ It is essential for dental professionals to routinely screen the patients and assess the role of diet in caries risk management. ${ }^{[13]}$ The present tech-savvy generation is governed by the use of apps for the easy of performing day-to-day basic tasks. Many of these apps aid individual behavioral change toward health and fitness. Previous study has been done to evaluate the effectiveness of android application for physical fitness and weight loss. ${ }^{[14]}$ As a technological initiative in the field of dentistry, "Cariometer" an android application has been developed by the authors in which day-to-day diet consumed is entered with the corresponding time of food consumption. This application is available for android phones and can be downloaded from the internet from Google App Store https://play.google.com/store/apps/ details?id=gowtham.example.com.dentalcare. The app gives a diet score based on the cumulative mean of the cariogenic score as given by Palmer et al. in 2010. ${ }^{[15]}$ Parents can enter the food consumed along with the time of consumption choosing from the list given in the app. The total dietary score is given by the app which is cumulated with addition of new data as and when it is entered during the day. The oral hygiene practice such as brushing rinsing and flossing is also entered which reverses the increase in score to zero. The application evaluates the dietary and oral hygiene practice of the child and graded as excellent, average, or bad. The application also gives an alarm when the dietary score is in the bad or average category and oral hygiene habit is not practiced. Cariometer also contains a feature where total score of the day and the cumulative score for the week or any customized date can be obtained. The report obtained can be mailed to the dentist who can advise on the dietary pattern and oral hygiene practice of the child. Thus, this app benefits both the child and parent in prevention of dental caries. Cariometer is in the process of obtaining patency from the Indian Patency Act with application no 201641035345.

To study the effectiveness of this application, a pilot study was conducted with the aim of monitoring the diet and oral hygiene habits. The null hypothesis of the present study states that the use of cariometer will have no improvement in the diet and oral hygiene habit.

\section{MATERIALS AND METHODS}

The ethical approval was obtained from the ethical committee board of Saveetha dental college (STP/SDMDS16PED3-A). This was a pilot study conducted in the Department of Pediatric and Preventive dentistry in Saveetha Dental College, Chennai. Parents of children in the age group of 2-6 years (mean: 5.5 years) were included in the study. A total of 150 children visiting Department of Pediatric and Preventive Dentistry of Saveetha Dental College for dental treatment between November 1, 2016 and January 31, 2017 were recruited to participate in the study based on convenience sampling. Parents of medically fit children were included. Parents using android phones were included in the study. Parents of all the children were asked about the dietary and oral hygiene habits and were introduced to cariometer application. They were asked to enter daily consumption of food as and when it was consumed by the child in the application for 7 days. Data of participants were included for analysis only when complete entry of food consumption and oral hygiene instruction were entered by the parents. Due to incomplete data obtained from some children, a final of 132 children were included in the study. All the children included for the study had similar cultural norms.

\section{Description of cariometer application}

Cariometer can be downloaded and installed in the smartphone operating on android system. In the first page of Cariometer, registration of each child can be completed with name and age. The parents can enter the food items by selecting from the list 
of different categories of food which includes: milk/cheese, beverage, sweet/chips/chocolate/ bubblegum, fresh and dry fruits/nuts/vegetables, rice/oats/cereals/wheat/bread, nonvegetarian side dish/rice, fresh juice/ice-cream/milkshake, and medication. The oral hygiene practice including rinsing after meals, brushing, and flossing can also be noted in the app.

The parents were asked to use cariometer app in which they had to enter the daily food consumed at the same time the food was consumed. Parents entered for the child during school hours by assuming the child has consumed food given by the parents during the lunch and/or break time. They were also asked to note the rinsing brushing or flossing after meals by selecting from the oral hygiene tab. Cariometer gives a dietary score every time the food and oral hygiene habit was entered. This score was cumulated over the day into a single score for the day. The app also gives pictorial representation and dietary reinforcements for the consumption [Figure 1]. Of healthy food after entering each food item. After consumption of all the meals for the day, parents were asked to mail the daily report which contained the dietary score and oral hygiene habit as given by the app. The parents continued using this application for 7 days. The mail report contained the rinsing habit post the use of the app as well as the brushing frequency for each day that the patient followed after using the app. All children were given dietary instructions and modifications in the diet to prevent caries post 7 days based on the dietary pattern that was followed by the children. These children using cariometer were further followed up to evaluate the effectiveness of the application in a long-term longitudinal study.

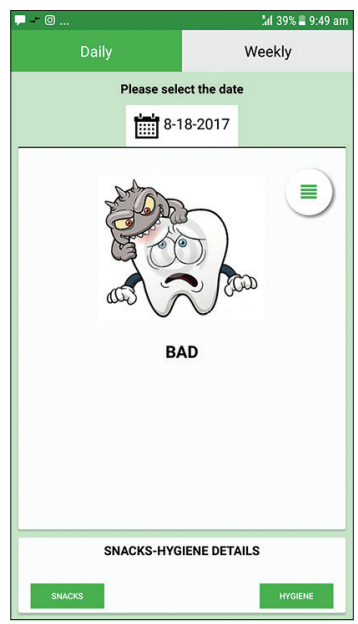

Figure 1: Pictorial representation of diet score as given by the app for the patient

\section{Statistical analysis}

The data obtained were recorded on a spread sheet and their respective mean and standard deviation (SD) were calculated. The adjusted mean was calculated by IBM SPSS statistics for Windows, version 22.0 (IBM Corp, Armont, NY, USA). These were the mean of the covariant and analysis of covariance (ANCOVA) was conducted for the dietary score obtained in the app at day 1 , day 4 , and day 7 . Paired $t$-test was conducted to compare the pre- and post-dietary score, the brushing and rinsing frequency. A statistical significance level was set at $P<0.05$.

\section{RESULTS}

Of the 150 participants, the data of 132 parents were considered for analysis. However, there was attrition of the sample in the present study. Three parents did not report at the end of 7 days due to disinterest in participating in the study, four parents missed entering data for 1 or 2 days and 11 parents did not enter data at the time of consumption of food. Due to incomplete data obtained from parents of 18 children, a final of 132 children were included in the study.

The Normality tests Kolmogorov-Smirnov and Shapiro-Wilks tests results reveal that variable (Diet Score) follows normal distribution. Therefore, to analyze the data parametric methods were applied. To compare the diet score between time points repeated measure ANCOVA test for repeated measures was used. To analyze the data SPSS (IBM SPSS Statistics for Windows, Version 22.0, Released 2013, IBM Corp. Armonk, NY, USA) were used. Significance level is fixed as $5 \%(\alpha=0.05)$.

The mean diet score on day 1 was 1.51 with SD of 0.244 . The mean diet score on day 4 was 0.83 with SD of 0.276 . The mean score on day 7 was 0.07 with SD of 0.143 [Table 1].

The ANCOVA showed a significant decrease in the dietary score obtained at the end of 7 days as compared to day 1 [Table 1 and Figure 2].

There was a significant improvement in the brushing frequency, with approximately $90 \%$ of children brushing twice a day after the use of the app [Table 2 and Figure 3]. None of the children rinsed after the meals before the use of the app. This was significantly increased to approximately $90 \%$ children rinsing after every meals [Table 3 and Figure 4]. 
Panchal, et al.: Cariometer: A click against caries

\begin{tabular}{|c|c|c|c|c|c|c|c|}
\hline Diet score & $n$ & Mean & SD & $1^{\text {st }}$ quartile & Median & $3^{\text {rd }}$ quartile & $P$ \\
\hline Day 1 & 132 & 1.51 & 0.244 & 1.4 & 1.5 & 1.7 & $<0.001$ \\
\hline Day 4 & 132 & 0.83 & 0.276 & 0.7 & 0.8 & 1.0 & $<0.001$ \\
\hline Day 7 & 132 & 0.07 & 0.143 & 0 & 0 & 0.1 & $<0.001$ \\
\hline
\end{tabular}

\begin{tabular}{|c|c|c|c|}
\hline & \multicolumn{3}{|c|}{ Brushing frequency: Day 1} \\
\hline & $\begin{array}{l}\text { Once, } \\
n(\%)\end{array}$ & $\begin{array}{l}\text { Twice, } \\
n(\%)\end{array}$ & $\begin{array}{l}\text { Total, } \\
n(\%)\end{array}$ \\
\hline \multicolumn{4}{|c|}{ Brushing frequency: Day 7} \\
\hline Once & $14(10.6)$ & 0 & $14(10.6)$ \\
\hline Twice & $118(89.4)$ & 0 & $118(89.4)$ \\
\hline Total & $132(100.0)$ & 0 & $132(100.0)$ \\
\hline
\end{tabular}

\begin{tabular}{|c|c|c|c|}
\hline & \multicolumn{3}{|c|}{ Rinsing after meals: Day 1} \\
\hline & No, $n(\%)$ & Yes, $n(\%)$ & Total, $\boldsymbol{n}(\%)$ \\
\hline \multicolumn{4}{|c|}{ Rinsing after meals: Day 7} \\
\hline No & $14(10.6)$ & 0 & $14(10.6)$ \\
\hline Yes & $118(89.4)$ & 0 & $118(89.4)$ \\
\hline Total & $132(100.0)$ & 0 & $132(100.0)$ \\
\hline
\end{tabular}

\section{DISCUSSION}

Technology has become a pivotal part of our day today system. With the introduction of android system, it has been bought to our fingertips. In this regard, cariometer has been developed to bring caries prevention to the finger tips.

Similar study have been done using mobile application to make a dietary assessment based on the type of food consumed showing the calorie intake, ${ }^{[16]}$ however no application measures the dietary score based on the weighted mean on the cariogenic risk of individual food items. This provides the uniqueness for the present study.

Caries risk assessment tool has been developed previously. American academy of pediatric dentistry has developed CAT to monitor the caries risk based on the exposure to fluoride, clinical findings, presence, or absence of caries. ${ }^{[17]}$

Another system used to assess the caries risk of the patient is cariogram, which was developed by Bratthall and Hänsel Petersson in the year 2004. This examines the caries risk based on the multifactorial nature of the dental caries. ${ }^{[18]}$

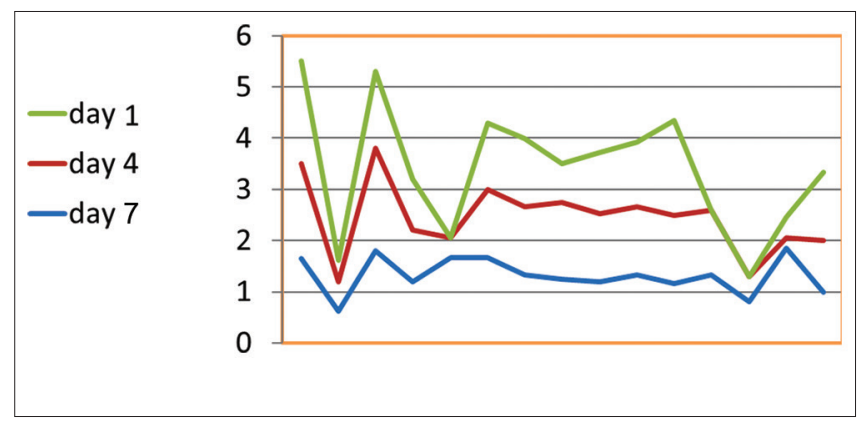

Figure 2: Comparison of 3 days diet score

These previous applications developed are however for professional use which cannot be used by the common man for the primary level of caries prevention. In this regard, to increase the cooperation and involvement of patients in caries prevention, this application was developed. Cariometer can be useful on day-to-day basis for caries prevention. The present study is the first of its kind done using android application estimating the caries risk based on the diet and oral hygiene. Diet counseling has always been the primordial level of caries prevention. ${ }^{[3,19]}$ In the regular diet counseling, physical presence of the dentist is not feasible as a constant reminder and reinforcement. However, with the introduction of the cariometer, this can be made possible thus increasing the efficacy of diet counseling. This application aids in prevention of the caries at the primordial level of development if started using from an early age.

Parents of children who participated in the study were the people who came for treatment. Hence, the knowledge of parents regarding oral health could be a decisive factor in usage of the app. This could have influenced the results. However, since the app is new initiative evaluation before and after the usage of the app with controlled follow-up is essential. Hence, this hospital-based study provides valuable information on the usage of application. There was a significant improvement in the dietary habits before and after the use of cariometer. This can be because cariometer allows a detailed accounting of the food consumed giving the cumulative cariogenic score for the corresponding diet consumed. Thus, giving the patient knowledge of the cariogenic food 


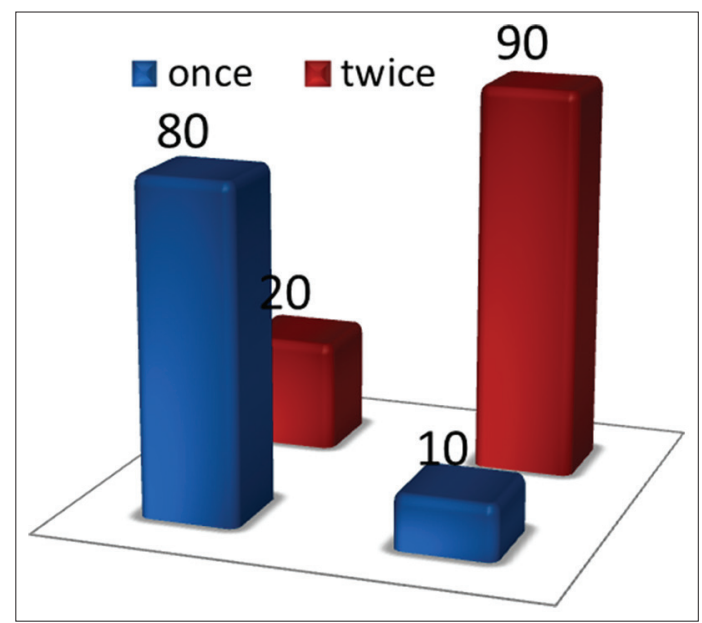

Figure 3: Comparison of brushing frequency pre and post the use of cariometer

consumption. Second, a pictorial representation of the cariogenic status of the teeth aids as an alert and helps in the maintenance of diet. There is also a feature which alerts the user if the cariogenic food consumption exceeds the optimal limit. This acts as a reminder to decrease the cariogenic dietary intake. Not only will it provide a check on the dietary intake, but also on the increase in the consumption of protective food. Increase in the consumption of protective food aids in increase in the good score, thereby acting as reinforcement. The diet diary followed in the app was according to article given by Palmer et al. ${ }^{[15]}$ A standard 7 days diet was followed as shown by previous studies. ${ }^{[20]}$

The significant improvement on the oral hygiene habit as shown by the patients can also be contributed to the recurrent alarm which acts as a reinforcement and a reminder to rinse and brush. This application also includes the effect of rinsing and other oral hygiene practices on the caries score further reinforcing oral hygiene habits. This promotes the development of basic cleaning measures among the patients. It allows the patients to monitor the diet daily as well as weekly. It provides a personalized caries management system for the patients.

According to a previous study by Damle et al., evaluating the effectiveness of supervised tooth brushing and oral health education on oral hygiene showed significant improvement in the oral hygiene habit. ${ }^{[6]}$ These results correlate with the present study showing improvement in the oral hygiene by reinforcing oral hygiene habits. Study by Nizel and Papas $^{[20]}$ shows the effectiveness of dietary counseling by maintaining diary on the prevention and control of

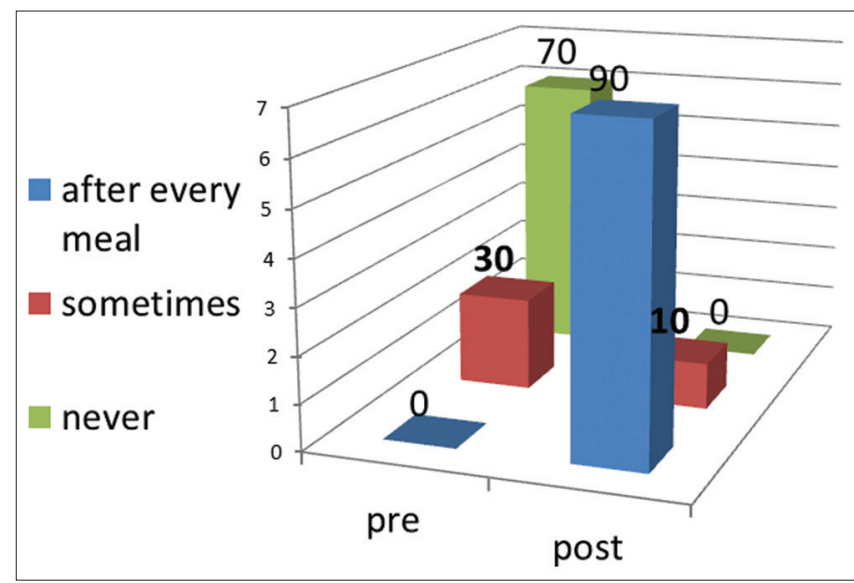

Figure 4: Comparison of rinsing after meals pre and post the use of cariometer

dental caries. These results coincide with the present study showing improvement in the dietary score by maintaining a proper dietary record.

Cariometer gives an idea for the caries risk the patient is subjected to. It does not include the multifactorial nature of dental caries and focus only on dietary and oral hygiene aspects. Additional supplemental chair side use of caries activity test can be beneficial to determine accurate caries risk. Cariometer is a preliminary step to bring caries prevention to the finger tips.

Cariometer encompasses certain limitations which were encountered during the duration of the study. The food list present needs to be updated incorporating the food items from all the cultural diversity. Furthermore, the person needs to be present all the time to reply to the mail, increasing the need for more manpower. This is in the process of being overcome by developing an autogenerated reply system which can send remarks to the parents based on the dietary score. Further, diet counseling can be obtained personally by the dentist on the dental visit. All the limitations are in the process to be overcome in the further version of application.

The present study uses the 7 days method to record the diet which will include the school going days as well as the holidays where the children tend to have increased snacking. However, a bias can be encountered in the present study while recording the diet consumed in school. This could be overcome in the further studies by encouraging the teacher to enter the food consumed by the children during the school hours. 
Another limitation of the present study is small sample size with lesser duration. Further continuation of the present study with long term follow-up, and larger sample size is needed to achieve the correct correlation and have more sustainable results. A randomized controlled trial comparing cariometer with the conventional diet diary will be ideal study design to be followed and obtaining more conclusive results. The present study is being continued in the institution with the randomized controlled trial design having a long-term follow-up. Furthermore, the application of present study in the population with varying cultural diversity, will aid to check vitality of the application to different population.

Cariometer successfully gives an approximate estimate of the caries risk based on the diet and reinforces oral hygiene habits. It is an attempt to utilize technology to every household to aid in the prevention of dental caries.

\section{CONCLUSION}

Cariometer is used to assess the quality of dietary habit and frequency of oral hygiene habits and also aid in its improvement by providing reinforcement using recent technological advances.

\section{Acknowledgment}

Acknowledging Mr. Gowtham Ashok for his assistance in the coding for the app.

\section{Financial support and sponsorship}

Nil.

\section{Conflicts of interest}

There are no conflicts of interest.

\section{REFERENCES}

1. Dixit A, Aruna DS, Sachdev V, Sharma A. Prevalence of dental caries and treatment needs among 3-5 year old preschool children in Narmada, Gujarat. IOSR J Dent Med Sci 2015;14:97-101.
2. Dye BA, Tan S, Smith V, Lewis BG, Barker LK, Thornton-Evans G, et al. Trends in oral health status: United States, 1988-1994 and 1999-2004. Vital Health Stat 11 2007;248:1-92.

3. Chour GV, Chour RG. Diet counselling - A primordial level of prevention of dental caries. IOSR J Dent Med Sci 2014;13:64-70.

4. Featherstone JD. Dental caries: A dynamic disease process. Aust Dent J 2008;53:286-91.

5. Stephan RM. Changes in hydrogen-ion concentration on tooth surface and in carious lesions. J Am Dent Assoc 1940;27:718-23.

6. Damle SG, Patil A, Jain S, Damle D, Chopal N. Effectiveness of supervised toothbrushing and oral health education in improving oral hygiene status and practices of urban and rural school children: A comparative study. J Int Soc Prev Community Dent 2014;4:175-81.

7. Cappelli DP, Mobley CC. Dental caries and associated risk factors. In: Prevention in Clinical Oral Health Care. $1^{\text {st }}$ ed. St. Louis, MO: Mosby Elsevier; 2008. p. 65-7.

8. Kant AK, Graubard BI, Schatzkin A. Dietary patterns predict mortality in a national cohort: The National Health Interview Surveys, 1987 and 1992. J Nutr 2004;134:1793-9.

9. Díaz-Garrido N, Lozano C, Giacaman RA. Frequency of sucrose exposure on the cariogenicity of a biofilm-caries model. Eur J Dent 2016;10:345-50.

10. Manju M, Prathyusha P, Joseph E, Kaul RB, Shanthraj SL, Sethi N, et al. Evaluation of the effect of three supplementary oral hygiene measures on salivary mutans streptococci levels in children: A randomized comparative clinical trial. Eur J Dent 2015;9:462-9.

11. Mobley C, Marshall TA, Milgrom P, Coldwell SE. The contribution of dietary factors to dental caries and disparities in caries. Acad Pediatr 2009;9:410-4.

12. Moyniham P, Lingstrom $P$, Rugg-Gunn AJ, Birkhed D. The role of Dietary control. In: Fejerskow O, Kidd E, editors. Dental Caries: The Disease and its Clinical Management. Oxford: Blackwell Munksgaard KJ; 2003. p. 222-41.

13. Karolinska I, Susanna A, Lars GP, Helena D, Soder B, Gunilla N, et al. Effect of combined caries- preventive methods: A systematic review of controlled clinical trials. Acta Odontol Scand 2004;62:163-9.

14. Laing BY, Mangione CM, Tseng CH, Leng M, Vaisberg E, Mahida M, et al. Effectiveness of a smartphone application for weight loss compared with usual care in overweight primary care patients: A randomized, controlled trial. Ann Intern Med 2014;161:S5-12.

15. Palmer CA, Kent R Jr. Loo CY, Hughes CV, Stutius E, Pradhan N, et al. Diet and caries-associated bacteria in severe early childhood caries. J Dent Res 2010;89:1224-9.

16. Pendergast FJ, Ridgers ND, Worsley A, McNaughton SA. Evaluation of a smartphone food diary application using objectively measured energy expenditure. Int J Behav Nutr Phys Act 2017;14:30.

17. Council on Clinical Affairs. Guidelines on caries risk assessment and management for infants and children and adolescent. AAPD 2002;37:15-6.

18. Bratthall D, Hänsel Petersson G. Cariogram - A multifactorial risk assessment model for a multifactorial disease. Community Dent Oral Epidemiol 2005;33:256-64.

19. Mirjalili N, Hassan M, Karbasi A, Farahman J. Comparing tap water rinse with tooth brushing and sugar free chewing gum: Investigating the validity of a popular belief. J Dent Oral Hyg 2014;6:22-5.

20. Nizel AE, Papas AS. Dietary counselling for the prevention and control of dental caries. In: Nutrition in Clinical Dentistry. $3^{\text {rd }}$ ed. Philadelphia: W.B. Saunders; 1989. p. 277-308. 